\title{
Continued Fraction Expansion of the Heinz Operator Mean
}

\author{
Kacem Belhroukia ${ }^{1}$, Salah Salhi ${ }^{2}$, Ali Kacha ${ }^{1, *}$ \\ ${ }^{1}$ Department of Mathematics, Science Faculty, Ibn Toufail University, Kenitra, Morocco \\ ${ }^{2}$ Department of Mathematics, Regional Center for Education and Training Professions, Errachidia, Morocco

\section{Email address:} \\ belhroukia.pc@gmail.com (K. Belhroukia), salhisalh@gmail.com (S. Salhi), ali.kacha@uit.ac.ma (A. Kacha) \\ *Corresponding author
}

\section{To cite this article:}

Kacem Belhroukia, Salah Salhi, Ali Kacha. Continued fraction expansion of the Heinz operator Mean. American Journal of Applied Mathematics. Vol. 8, No. 9, 2020, pp. 311-318. doi: 10.11648/j.ajam.20200806.13

Received: November 6, 2019; Accepted: December 20, 2019; Published: December 6, 2020

\begin{abstract}
We recall that means arise in various contexts and contribute to solving many scientific problems. The aim of the present paper is to give a continued fraction expansion of the Heinz operator mean for two positive definite matrices. We note that the direct calculation of the Heinz operator mean proves difficult by the appearance of rational exponents of matrices. The main motivation of this work is to overcome these difficulties and to present a practical and efficient method for this calculation. We use the matrix continued fraction algorithm. At the end of our paper, we deduce a continued fraction representation of the symmetric operator entropy.
\end{abstract}

Keywords: Continued Fraction, Positive Definite Matrix, Heinz Operator Mean

\section{Introduction and Motivation}

The basic idea of the continued fraction theory over real numbers is to give an approximation of various real numbers by the rational. A continued fraction is an expression obtained through an iterative process of representing a number as the sum of its integer part and the reciprocal, and so on. One of the main reasons why continued fractions are so useful in computation is that they often provide representation for transcendental functions that are much more generally valid than the classical representation by, say, the power series. Further; in the convergent case, the continued fractions expansions have the advantage that they converge more rapidly than other numerical algorithms.

Recently, the extension of continued fractions theory from real numbers to the matrix case has seen several development and interesting applications, ([4-8]). Since calculations involving matrix valued functions with matrix arguments are feasible with large computers, it will be an interesting attempt to develop such matrix theory. The real case is relatively well studied in the literature ([9-10]). However, in contrast to the theoretical importance, one can find in mathematical literature only a few results on the continued fractions with matrix arguments ([12-16]).

The theory of operator means for positive (bounded linear) operators on an Hilbert space was initiated by T. Ando and established by him and F. Kubo in connection with Lowner's theory for the operator monotone functions, see [1-3].

We notice that one remarkable mean, which interpolates between the geometric and the arithmetic means is so called Heinz mean $H_{\alpha}$, defined for two positive real numbers $a, b$ by ([5]).

$$
H_{\alpha}(a, b)=\frac{a^{\alpha} b^{1-\alpha}+a^{1-\alpha} b^{\alpha}}{2},
$$

where $\alpha$ is a given real number such that $0 \leq \alpha \leq 1$.

It is obvious that $\sqrt{a b} \leq H_{\alpha}(a, b) \leq(a+b) / 2, \alpha \in[0,1]$. The function $H_{\alpha}$ is symmetric about $\alpha=\frac{1}{2}$. We also note that

$$
H_{0}(a, b)=H_{1}(a, b)=\frac{a+b}{2} \text { and } H_{1 / 2}(a, b)=\sqrt{a b} .
$$

In the present paper, we give a continued fraction representation of the Heinz operator mean for two positive definite matrices $A$ and $B$. We recall that this operator is defined by

$$
H_{\alpha}(A, B)=A^{\frac{1}{2}} \frac{\left(A^{\frac{-1}{2}} B A^{\frac{-1}{2}}\right)^{\alpha}+\left(A^{\frac{-1}{2}} B A^{\frac{-1}{2}}\right)^{1-\alpha}}{2} A^{\frac{1}{2}} .
$$


Therefor, We also recall that the symmetric operator entropy for two positive definite matrices $A$ and $B$ is defined by

$$
S T_{\alpha}(A, B)=\frac{1}{\alpha}\left(H_{\alpha}(A, B)-\frac{A+B}{2}\right) .
$$

\section{Definitions and Notations}

The functions of matrix arguments play a widespreased role in science and engineering, with applications areas from nuclear magnetic resonance [1]. So for scalar polynomial $p(z)=\sum_{i=0}^{k} a_{i} z^{i}$ gives rise to a matrix polynomial with scalar coefficients by simply substitution $A^{i}$ for $z^{i}$ :

$$
P(A)=\sum_{i=0}^{k} a_{i} A^{i}
$$

More generally, for a function $f$ with a series representation on an open disk containing the eigenvalues of $A$, we are able to define the matrix function $f(A)$ via the Taylor series for $f$, see $[10]$.

Alternatively, given a function $f$ that is analytic inside a closed contour $\Gamma$ which enclose the eigenvalues of $A, f(A)$ can be defined, by analogy with Cauchy's integral theorem by

$$
f(A)=\frac{1}{2 \pi i} \int_{\Gamma} f(z)(z I-A)^{-1} d z .
$$

The definition is known as the matrix version of Cauchy's integral theorem. Let $\mathcal{M}_{m}$ be the algebra of real square matrices, we now mention an important result of matrix functions.

Let $A \in \mathcal{M}_{m}, A$ is said to be positive semi-definite (resp. positive definite) if $A$ is symmetric and

$$
\begin{gathered}
\forall x \in \mathbb{R}^{m}, \\
\left.<A x, x>\geq 0 \text { (resp. } \forall x \in \mathbb{R}^{m} x \neq 0,<A x, x>>0\right) .
\end{gathered}
$$

where $<., .>$ denotes the standard scalar product of $\mathbb{R}^{m}$ defined by

$$
\begin{gathered}
x=\left(x_{1}, \ldots, x_{m}\right), \\
y=\left(y_{1}, \ldots, y_{m}\right) \in \mathbb{R}^{m}:<x, y>=\sum_{i=1}^{m} x_{i} y_{i} .
\end{gathered}
$$

We observe that positive semi-definiteness induces a partial ordering on the space of symmetric matrices. Henceforth, whenever we say that $A \in \mathcal{M}_{m}$ is positive semi-definite (or positive definite), it will be assumed that $A$ is symmetric.

For any $A, B \in \mathcal{M}_{m}$ with $B$ invertible, we write $\frac{A}{B}=$ $B^{-1} A$, in particular, if $A=I$, where $I$ is the $m^{t h}$ order identity matrix, then $\frac{I}{B}=B^{-1}$. It is clear that for any invertible matrix $C$, we have

$$
\frac{C A}{C B}=\frac{A}{B} \neq \frac{A C}{B C}
$$

Definition 2.1. Let $\left(A_{n}\right)_{n>0},\left(B_{n}\right)_{n>0}$ be two nonzero sequences of $\mathcal{M}_{m}$. The continued fraction of $\left(A_{n}\right)$ and $\left(B_{n}\right)$ denoted by $K\left(B_{n} / A_{n}\right)$ is the quantity

$$
A_{0}+\frac{B_{1}}{A_{1}+\frac{B_{2}}{A_{2}+\cdots}}=\left[A_{0} ; \frac{B_{1}}{A_{1}}, \frac{B_{2}}{A_{2}}, \cdots\right] .
$$

Sometimes, we use briefly the notation $\left[A_{0} ; \frac{B_{n}}{A_{n}}\right]_{n=1}^{+\infty}$. The fractions $\frac{B_{n}}{A_{n}}$ and $\frac{P_{n}}{Q_{n}}=\left[A_{0} ; \frac{B_{k}}{A_{k}}\right]_{k=1}^{n}$ are called, respectively, the $n^{\text {th }}$ partial quotient and the $n^{\text {th }}$ convergent of the continued fraction $K\left(B_{n} / A_{n}\right)$.

When $B_{n}=I$ for all $n \geq 1$, then $K\left(I / A_{n}\right)$ is called a simple continued fraction.

We now introduce some topological notion of continued fractions with matrix arguments. Let $A \in \mathcal{M}_{m}$, we put

$$
\|A\|=\sup _{x \neq 0}\left\{\frac{\|A x\|}{\|x\|}\right\}=\sup _{\|x\|=1}\{\|A x\|\} .
$$

Let $\left(A_{n}\right)$ be a sequence of matrices in $\mathcal{M}_{m}$. We say that $\left(A_{n}\right)$ converges in $\mathcal{M}_{m}$ if there exists a matrix $A \in \mathcal{M}_{m}$ such that $\lim _{n \rightarrow+\infty}\left\|A_{n}-A\right\|=0$.

The continued fraction $K\left(B_{n} / A_{n}\right)$ converges in $\mathcal{M}_{m}$ if the sequence $\left(F_{n}\right)=\left(\frac{P_{n}}{Q_{n}}\right)=\left(Q_{n}^{-1} P_{n}\right)$ converges in $\mathcal{M}_{m}$ in the sense that there exists a matrix $F \in \mathcal{M}_{m}$ such that $\lim _{n \rightarrow+\infty}\left\|F_{n}-F\right\|=0$. In this case, we note

$$
F=\left[A_{0} ; \frac{B_{n}}{A_{n}}\right]_{n=1}^{+\infty}
$$

We note that the evaluation of $n^{t h}$ convergent according to the Definition 1 is not practical because we have to repeat inverse matrix. The following proposition gives an adequate method to calculate $K\left(B_{n} / A_{n}\right)$.

Proposition 2.1. The elements $\left(P_{n}\right)_{n \geq-1}$ and $\left(Q_{n}\right)_{n \geq-1}$ of the $n^{\text {th }}$ convergent of $K\left(B_{n} / A_{n}\right)$ are given by the relationships

$$
\left\{\begin{array}{cc}
P_{-1}=I, & P_{0}=A_{0} \\
Q_{-1}=0, & Q_{0}=I
\end{array}\right.
$$

and

$$
\left\{\begin{array}{c}
P_{n}=A_{n} P_{n-1}+B_{n} P_{n-2} \\
Q_{n}=A_{n} Q_{n-1}+B_{n} Q_{n-2}
\end{array}, \quad n \geq 1 .\right.
$$

Proof. We prove it by induction.

The proof of the next Proposition is elementary and we left it to the reader.

Proposition 2.2. For any two matrices $C$ and $D$ with $C$ invertible, we have

$$
C\left[A_{0} ; \frac{B_{k}}{A_{k}}\right]_{k=1}^{n} D=\left[C A_{0} D ; \frac{B_{1} D}{A_{1} C^{-1}} ; \frac{B_{2} C^{-1}}{A_{2}} ; \frac{B_{k}}{A_{k}}\right]_{k=3}^{n} .
$$

Definition 2.2. Let $\left\{A_{n}\right\},\left\{B_{n}\right\},\left\{C_{n}\right\}$, and $\left\{D_{n}\right\}$ be four 
sequences of matrices. We say that the continued fractions $K\left(B_{n} / A_{n}\right)$ and $K\left(D_{n} / C_{n}\right)$ are equivalent if we have $F_{n}=$ $G_{n}$ for all $n \geq 1$, where $F_{n}$ and $G_{n}$ are the $n^{\text {th }}$ convergent of $K\left(B_{n} / A_{n}\right)$ and $K\left(D_{n} / C_{n}\right)$ respectively.

In order to simplify the statement on some partial quotients of continued fractions with matrix arguments, we need the following proposition which is an example of equivalent continued fractions.

Proposition 2.3 (14). Let $\left[A_{0} ; \frac{B_{k}}{A_{k}}\right]_{k=1}^{+\infty}$ be a given continued fraction. Then we have

$$
\frac{P_{n}}{Q_{n}}=\left[A_{0} ; \frac{B_{k}}{A_{k}}\right]_{k=1}^{n}=\left[A_{0} ; \frac{X_{k} B_{k} X_{k-2}^{-1}}{X_{k} A_{k} X_{k-1}^{-1}}\right]_{k=1}^{n}
$$

where $X_{-1}=X_{0}=I$ and $X_{1}, X_{2}, \cdots \cdot \cdot, X_{n}$ are arbitrary invertible matrices.

Proof. Let $\frac{P_{n}}{Q_{n}}$ and $\frac{\widetilde{P}_{n}}{\widetilde{Q}_{n}}$ be the $n^{\text {th }}$ convergent of the continued fractions $\left[A_{0} ; \frac{B_{k}}{A_{k}}\right]_{k=1}^{+\infty}$ and $\left[A_{0} ; \frac{X_{k} B_{k} X_{k-2}^{-1}}{X_{k} A_{k} X_{k-1}^{-1}}\right]_{k=1}^{+\infty}$ respectively. By proposition 2 , for all $n \geq 1$, we can write

$$
\widetilde{P}_{n}=X_{n} A_{n} X_{n-1}^{-1} \widetilde{P}_{n-1}+X_{n} B_{n} X_{n-2}^{-1} \widetilde{P}_{n-2},
$$

which is equivalent to

$$
X_{n}^{-1} \widetilde{P}_{n}=A_{n}\left(A_{n} X_{n-1}^{-1} \widetilde{P}_{n-1}\right)+B_{n}\left(X_{n-2}^{-1} \widetilde{P}_{n-2}\right)
$$

Theorem 3.1. Let $A \in \mathcal{M}_{m}$ be a positive definite matrix and $\alpha$ a positive real number such that $0<\alpha<1$. If we put $A^{\alpha}=\left[I ; \frac{I}{A_{k}}\right]_{k=1}^{+\infty}, A^{1-\alpha}=\left[I ; \frac{I}{\widetilde{A}_{k}}\right]_{k=1}^{+\infty}$ and $\varphi(A)=\frac{I-A}{I+A}$ then, the continued fraction expansions of $\left(A^{\alpha}+A^{1-\alpha}\right)$ is given by

$$
A^{\alpha}+A^{1-\alpha}=\left[2 I ; \frac{A_{1}+\widetilde{A}_{1}}{A_{1} \widetilde{A}_{1}}, \frac{A_{1}\left(A_{1}+I\right)+\widetilde{A}_{1}\left(\widetilde{A}_{1} \widetilde{A}_{2}+I\right)}{A_{1} A_{2} \widetilde{A}_{2}+\widetilde{A}_{1} \widetilde{A}_{2} A_{2}+A_{2}+\widetilde{A}_{2}}, \frac{\left(A_{1} \widetilde{A}_{1}\right)^{-1} E_{2} F_{3}}{E_{3}-F_{3}}, \frac{E_{n-1} F_{n}}{E_{n}-F_{n}}\right]_{n=4}^{+\infty}
$$

where

$$
\left\{\begin{array}{l}
A_{1}=\frac{-I-\alpha \varphi(A)}{2 \alpha \varphi(A)}, \\
A_{2 k}=\frac{-2 \alpha\left(\alpha^{2}-2^{2}\right) \cdots\left(\alpha^{2}-(2 k-2)^{2}\right)}{\left(\alpha^{2}-1\right) \cdots\left(\alpha^{2}-(2 k-1)^{2}\right)}(4 k-1) I, k \geq 1 \\
A_{2 k+1}=\frac{-\left(\alpha^{2}-1\right) \cdots\left(\alpha^{2}-(2 k-1)^{2}\right)}{2 \alpha\left(\alpha^{2}-2^{2}\right) \cdots\left(\alpha^{2}-4 k^{2}\right)}(4 k+1) \varphi^{2}(A), k \geq 1 .
\end{array}\right.
$$

For all $n \geq 1$, the expression of $\widetilde{A}_{n}$ is obtained from that of $A_{n}$ by replacing $\alpha$ by $(1-\alpha)$, with

$$
\left\{\begin{array}{l}
E_{n}=Q_{n}(\alpha) Q_{n}(1-\alpha)\left(Q_{n-2}(\alpha) Q_{n-1}(\alpha)+Q_{n-2}(1-\alpha) Q_{n-1}(1-\alpha)\right), \\
F_{n}=Q_{n-2}(\alpha) Q_{n-2}(1-\alpha)\left(Q_{n-1}(\alpha) Q_{n}(\alpha)+Q_{n-1}(1-\alpha) Q_{n}(1-\alpha)\right) .
\end{array}\right.
$$

The matrices $Q_{n}(\alpha)$ (resp. $Q_{n}(1-\alpha)$ are the denominators of the $n^{t h}$ convergent of $A^{\alpha}$ (resp. $A^{1-\alpha}$ ) which are defined by

$$
\left\{\begin{array}{l}
Q_{-1}(\alpha)=0, Q_{0}(\alpha)=I \\
Q_{-1}(1-\alpha)=0, Q_{0}(1-\alpha)=I
\end{array}, \quad\left\{\begin{array}{l}
Q_{n}(\alpha)=A_{n} Q_{n-1}(\alpha)+Q_{n-2}(\alpha) \\
Q_{n}(1-\alpha)=\widetilde{A}_{n} Q_{n-1}(1-\alpha)+Q_{n-2}(1-\alpha)
\end{array} .\right.\right.
$$

In order to prove Theorem 1 , we begin by studying the real case.
This last result joined to the initial conditions prove that for all $n \geq 1, X_{n-1}^{-1} \widetilde{P}_{n}=P_{n}$. A similar result can be obtained for $Q_{n}$. Consequently, both continued fractions have the same

\section{Main Results}

This section is devoted to give a continued fraction and $\alpha$ is a real number such that $0<\alpha<1$.

Definition 3.1. Let $A$ be a positive definite matrix in $\mathcal{M}_{m}, X \in \mathcal{M}_{m}$ and $\alpha$ a real number such that $0<\alpha<1$. We define the matrix $A^{\alpha}$ by the formulae

$$
A^{\alpha}=\exp (\alpha \log A),
$$

where "exp" is the matrix exponential given by the series

$$
\exp (X)=\sum_{n=0}^{+\infty} \frac{X^{n}}{n !}
$$

and "log" is the logarithm defined, for example by

$$
\log A=-2 \sum_{n=0}^{+\infty} \frac{1}{2 n+1}\left(\frac{I-A}{I+A}\right)^{2 n+1} .
$$




\subsection{The Real Case}

We begin by giving some lemmas concerning the real continued fraction which are important in the sequel. The following lemma characterizes equivalence of continued fractions.

Lemma 3.2. [9] Let $\left(r_{n}\right)$ be a non-zero sequence of real numbers. The continued fractions

$$
\left[a_{0} ; \frac{r_{1} b_{1}}{r_{1} a_{1}}, \frac{r_{2} r_{1} b_{2}}{r_{2} a_{2}}, \cdots, \frac{r_{n} r_{n-1} b_{n}}{r_{n} a_{n}}, \cdots\right] \text { and }\left[a_{0} ; \frac{b_{1}}{a_{1}}, \frac{b_{2}}{a_{2}}, \cdots, \frac{b_{n}}{a_{n}}, \cdots\right]
$$

are equivalent.

We now give a lemma which expresses the $n^{\text {th }}$ convergent for the sum of two continued fractions.

Lemma 3.3. [13] Let $C$ and $D$ be two real continued fractions which are defined by

$$
C=\left[c_{0} ; \frac{1}{c_{1}}, \frac{1}{c_{n}}\right]_{n=2}^{+\infty}, D=\left[d_{0} ; \frac{1}{d_{1}}, \frac{1}{d_{n}}\right]_{n=2}^{+\infty}
$$

where $c_{k}$ and $d_{k}$ are non-zero real numbers for $k \geq 1$. If we put

$$
C_{n}=\left[c_{0} ; \frac{1}{c_{1}}, \frac{1}{c_{2}}, \cdots, \frac{1}{c_{n}}\right]=\frac{{ }^{c} p_{n}}{{ }^{c} q_{n}}, \text { and } D_{n}=\left[d_{0} ; \frac{1}{d_{1}}, \frac{1}{d_{2}}, \cdots, \frac{1}{d_{n}}\right]=\frac{{ }^{d} p_{n}}{{ }^{d} q_{n}},
$$

then, for all $n \geq 1$, we have

$$
C_{n}+D_{n}=\left[c_{0}+d_{0} ; \frac{c_{1}+d_{1}}{c_{1} d_{1}}, \frac{c_{1} d_{1} f_{2}}{e_{2}-f_{2}}, \frac{e_{2} f_{3}}{e_{3}-f_{3}}, \cdots, \frac{e_{n-1} f_{n}}{e_{n}-f_{n}}\right]
$$

where

$$
\left\{\begin{array}{l}
e_{n}={ }^{c} q_{n}{ }^{d} q_{n}\left({ }^{c} q_{n-2}{ }^{c} q_{n-1}+{ }^{d} q_{n-2}{ }^{d} q_{n-1}\right) \\
f_{n}={ }^{c} q_{n-2}{ }^{d} q_{n-2}\left({ }^{c} q_{n-1}{ }^{c} q_{n}+{ }^{d} q_{n-1}{ }^{d} q_{n}\right)
\end{array}\right.
$$

The following Lemma gives two equivalent continued fraction expansions of $\lambda^{\alpha}$, where $\lambda$ and $\alpha$ are two strictly positive real numbers.

Lemma 3.4. i) Let $\lambda$ and $\alpha$ be two positive real numbers, $\varphi(\lambda)=\frac{1-\lambda}{1+\lambda}$. The continued fraction expansions of $\lambda^{\alpha}$ is

$$
\lambda^{\alpha}=\left[1 ; \frac{2 \alpha \varphi(\lambda)}{-1-\alpha \varphi(\lambda)}, \frac{\left(\alpha^{2}-(k-1)^{2}\right) \varphi^{2}(\lambda)}{-(2 k-1)}\right]_{k=2}^{+\infty}
$$

ii) If we put

$$
\left\{\begin{array} { l } 
{ b _ { 1 } = 2 \alpha \varphi ( r ) } \\
{ a _ { 1 } = - 1 - \alpha \varphi ( r ) }
\end{array} \quad \text { and } \left\{\begin{array}{l}
b_{k}=\left(\alpha^{2}-(k-1)^{2}\right) \varphi^{2}(r) \\
a_{k}=-(2 k-1)
\end{array}, k \geq 2\right.\right.
$$

then, the simple continued fraction of $\lambda^{\alpha}$ is given by

$$
\lambda^{\alpha}=\left[1 ; \frac{1}{a_{1}^{*}}, \frac{1}{a_{2}^{*}}, \cdots, \frac{1}{a_{k}^{*}}, \cdots\right]
$$

where

$$
\left\{\begin{array}{l}
a_{1}^{*}=\frac{a_{1}}{b_{1}}=\frac{-1-\alpha \varphi(\lambda)}{2 \alpha \varphi(\lambda)} \text { and for } k \geq 1, \\
a_{2 k}^{*}=\frac{b_{1} b_{3} \cdots b_{2 k-1}}{b_{2} b_{4} \cdots b_{2 k}} a_{2 k}=\frac{-2 \alpha\left(\alpha^{2}-2^{2}\right) \cdots\left(\alpha^{2}-(2 k-2)^{2}\right)}{\left(\alpha^{2}-1\right) \cdots\left(\alpha^{2}-(2 k-1)^{2}\right)}(4 k-1), \\
a_{2 k+1}^{*}=\frac{b_{2} b_{4} \cdots b_{2 k}}{b_{1} b_{3} \cdots b_{2 k+1}} a_{2 k+1}=\frac{-\left(\alpha^{2}-1\right) \cdots\left(\alpha^{2}-(2 k-1)^{2}\right)}{2 \alpha\left(\alpha^{2}-2^{2}\right) \cdots\left(\alpha^{2}-(2 k)^{2}\right)}(4 k+1) \varphi^{2}(\lambda) .
\end{array}\right.
$$

Proof. i) See [15].

ii) We prove it by appropriate iteration and by applying Lemma 1.

Remark 1. The continued fraction expansion of $\lambda^{1-\alpha}$ is obtained by substituting $\alpha$ by $(1-\alpha)$ in the previous formulas of $\lambda^{\alpha}$. 
So, we define

$$
\lambda^{1-\alpha}=\left[1 ; \frac{1}{\widetilde{a}_{1}^{*}}, \frac{1}{\widetilde{a}_{2}^{*}}, \cdots, \frac{1}{\widetilde{a}_{k}^{*}}, \cdots\right] .
$$

The next Theorem is a real version of the previous Theorem 1.

Theorem 3.5. With the same notations as bellow, Let $\lambda$ and $\alpha$ be two real numbers such that $\lambda>0$ and $0<\alpha<1$. A continued fraction expansion of the real $\left(\lambda^{\alpha}+\lambda^{1-\alpha}\right)$ is given by

$$
\lambda^{\alpha}+\lambda^{1-\alpha}=\left[2 ; \frac{a_{1}^{*}+\widetilde{a}_{1}^{*}}{a_{1}^{*} \widetilde{a}_{1}^{*}}, \frac{a_{1}^{*}\left(\widetilde{a}_{1}^{*}+1\right)+\widetilde{a}_{1}^{*}\left(\widetilde{a}_{2}^{*} \widetilde{a}_{1}^{*}+1\right)}{a_{1}^{*} a_{2}^{*} \widetilde{a}_{2}^{*}+\widetilde{a}_{1}^{*} \widetilde{a}_{2}^{*} a_{2}^{*}+a_{2}^{*}+\widetilde{a}_{2}^{*}}, \frac{\left(a_{1}^{*} \widetilde{a}_{1}^{*}\right)^{-1} e_{2} f_{3}}{e_{3}-f_{3}}, \cdots, \frac{e_{n-1} f_{n}}{e_{n}-f_{n}}\right]_{n=4}^{+\infty},
$$

where

$$
\left\{\begin{array}{l}
e_{n}=q_{n}(\alpha) q_{n}(1-\alpha)\left(q_{n-2}(\alpha) q_{n-1}(\alpha)+q_{n-2}(1-\alpha) q_{n-1}(1-\alpha)\right) \\
f_{n}=q_{n-2}(\alpha) q_{n-2}(1-\alpha)\left(q_{n-1}(\alpha) q_{n}(\alpha)+q_{n-1}(1-\alpha) q_{n}(1-\alpha)\right)
\end{array}\right.
$$

and $q_{n}(\alpha)$ (resp. $q_{n}(1-\alpha)$ is the denominator of the $n^{\text {th }}$ convergent of $\lambda^{\alpha}$ (resp. $\lambda^{1-\alpha}$ ). They are defined by

$$
\left\{\begin{array}{c}
q_{-1}(\alpha)=0, q_{0}(\alpha)=1 \\
q_{n}(\alpha)=a_{n}^{*} q_{n-1}(\alpha)+q_{n-2}(\alpha)
\end{array}, \quad\left\{\begin{array}{c}
q_{-1}(1-\alpha)=0, q_{0}(1-\alpha)=1 . \\
q_{n}(1-\alpha)=\widetilde{a}_{n}^{*} q_{n-1}(1-\alpha)+q_{n-2}(1-\alpha) .
\end{array}\right.\right.
$$

Proof. According to Lemma 3.4, we have

$$
\lambda^{\alpha}=\left[1 ; \frac{1}{a_{k}^{*}}\right]_{k=1}^{+\infty} \text { and } \lambda^{1-\alpha}=\left[1 ; \frac{1}{\widetilde{a}_{k}^{*}}\right]_{k=1}^{+\infty}
$$

Then, we apply Lemma 3.3 to complete the proof.

\section{Proof of Theorem 3.1 .}

Let $A \in \mathcal{M}_{m}$ be a positive definite matrix. Then there exists an invertible matrix $X$ such that $A=X D X X^{-1}$ where $D=$ $\left(\lambda_{1}, \lambda_{2}, \cdot \cdot \lambda_{m}\right)$ and $\lambda_{i}>0$, for all $1 \leq i<m$.

As the function $z \rightarrow z^{\alpha}$ is analytic in the open interval $\mathbb{R}_{+}^{*}$, then we get

$$
A^{\alpha}=X D^{\alpha} X^{-1}=X \operatorname{diag}\left(\lambda_{1}^{\alpha}, \lambda_{2}^{\alpha}, \cdots, \lambda_{m}^{\alpha}\right) X^{-1} .
$$

Therefore,

$$
A^{\alpha}+A^{1-\alpha}=X\left(D^{\alpha}+D^{1-\alpha}\right) X^{-1} .
$$

Let us define the sequences $\left(P_{n}\right)$ and $\left(Q_{n}\right)$ (the elements of the $n^{\text {th }}$ convergent of $\left.\left(D^{\alpha}+D^{1-\alpha}\right)\right)$ by

$$
\left\{\begin{array}{l}
P_{-1}=I, \quad P_{0}=2 I, \quad P_{1}=2 D_{1} \widetilde{D}_{1}+\left(D_{1}+\widetilde{D}_{1}\right), \quad P_{2}=\left(E_{2}^{\prime}-F_{2}^{\prime}\right) P_{1}+2 D_{2} \widetilde{D}_{2} F_{2}^{\prime} \\
Q_{-1}=0, \quad Q_{0}=I, \quad Q_{1}=D_{1} \widetilde{D}_{1}, \quad Q_{2}=\left(E_{2}^{\prime}-F_{2}^{\prime}\right) Q_{1}+D_{2} \widetilde{D}_{2} F_{2}^{\prime}
\end{array}\right.
$$

and for $n \geq 3$,

$$
\begin{gathered}
\left\{\begin{array}{c}
P_{n}=\left(E_{n}^{\prime}-F_{n}^{\prime}\right) P_{n-1}+E_{n-1}^{\prime} F_{n}^{\prime} P_{n-2} \\
Q_{n}=\left(E_{n}^{\prime}-F_{n}^{\prime}\right) Q_{n-1}+E_{n-1}^{\prime} F_{n}^{\prime} Q_{n-2}
\end{array}\right. \\
\left\{\begin{array}{c}
E_{n}^{\prime}=Q_{n}(\alpha) Q_{n}(1-\alpha)\left(Q_{n-2}(\alpha) Q_{n-1}(\alpha)+Q_{n-2}(1-\alpha) Q_{n-1}(1-\alpha)\right), \\
F_{n}^{\prime}=Q_{n-2}(\alpha) Q_{n-2}(1-\alpha)\left(Q_{n-1}(\alpha) Q_{n}(\alpha)+Q_{n-1}(1-\alpha) Q_{n}(1-\alpha)\right) .
\end{array}\right.
\end{gathered}
$$

The matrices $Q_{n}(\alpha)$ (resp. $Q_{n}(1-\alpha)$ ) are the denominators of the $n^{\text {th }}$ convergent of $D^{\alpha}$ (resp. $D^{1-\alpha}$ ) which are defined by

$$
\left\{\begin{array}{l}
Q_{0}(\alpha)=0, Q_{1}(\alpha)=D_{1}, \quad Q_{n}(\alpha)=D_{n} Q_{n-1}(\alpha)+Q_{n-2}(\alpha), \\
Q_{0}(1-\alpha)=0, \quad Q_{1}(1-\alpha)=\widetilde{D}_{1}, Q_{n}(1-\alpha)=\widetilde{D}_{n} Q_{n-1}(1-\alpha)+Q_{n-2}(1-\alpha) .
\end{array}\right.
$$


We recall that

$$
\left\{\begin{array}{l}
D_{1}=\frac{-I-\alpha \varphi(D)}{2 \alpha \varphi(D)}, \\
D_{2 k}=\frac{-2 \alpha\left(\alpha^{2}-2^{2}\right) \cdots\left(\alpha^{2}-(2 k-2)^{2}\right)}{\left(\alpha^{2}-1\right) \cdots\left(\alpha^{2}-(2 k-1)^{2}\right)}(4 k-1) I, k \geq 1, \\
D_{2 k+1}=\frac{-\left(\alpha^{2}-1\right) \cdots\left(\alpha^{2}-(2 k-1)^{2}\right)}{2 \alpha\left(\alpha^{2}-2^{2}\right) \cdots\left(\alpha^{2}-4 k^{2}\right)}(4 k+1) \varphi^{2}(D), k \geq 1 .
\end{array}\right.
$$

We see that $P_{n}, Q_{n}, E_{n}^{\prime}$ and $F_{n}^{\prime}$ are diagonal matrices, we put

$$
\left\{\begin{array}{l}
P_{n}=\operatorname{diag}\left(p_{n}^{1}, p_{n}^{2}, \cdots, p_{n}^{m}\right) \\
Q_{n}=\operatorname{diag}\left(q_{n}^{1}, q_{n}^{2}, \cdots, q_{n}^{m}\right)
\end{array} \quad, \quad\left\{\begin{array}{c}
E_{n}^{\prime}=\operatorname{diag}\left(e_{n}^{1}, e_{n}^{2}, \cdots, e_{n}^{m}\right) \\
F_{n}^{\prime}=\operatorname{diag}\left(f_{n}^{1}, f_{n}^{2}, \cdots, f_{n}^{m}\right) .
\end{array}\right.\right.
$$

We obtain for each $1 \leq i \leq m$,

$$
\begin{gathered}
\left\{\begin{array}{c}
p_{-1}^{i}=1, p_{0}^{i}=2, p_{1}^{i}=2 a_{i 1}^{*} \widetilde{a}_{i 1}+a_{i 1}^{*} \widetilde{a}_{i 1}^{*} \\
q_{-1}^{i}=0, q_{0}^{i}=1, q_{1}^{i}=\left(a_{i 1}^{*} \widetilde{a}_{i 1}^{*}\right)
\end{array}\right. \\
\left\{\begin{array}{c}
p_{2}^{i}=\left(e_{2}^{i}-f_{2}^{i}\right) p_{1}^{i}+2\left(a_{i 1}^{*} \widetilde{a}_{i 1}^{*}\right) f_{2}^{i} \\
q_{2}^{i}=\left(e_{2}^{i}-f_{2}^{i}\right) q_{1}^{i}+\left(a_{i 1}^{*} \widetilde{a}_{i 1}^{*}\right) f_{2}^{i}
\end{array}\right.
\end{gathered}
$$

and for $n \geq 3$, we have

$$
\begin{gathered}
\left\{\begin{array}{c}
p_{n}^{i}=\left(e_{n}^{i}-f_{n}^{i}\right) p_{n-1}^{i}+e_{n-1}^{i} f_{n-2}^{i} p_{n-2}^{i} \\
q_{n}^{i}=\left(e_{n}^{i}-f_{n}^{i}\right) q_{n-1}^{i}+e_{n-1}^{i} f_{n}^{i} q_{n-2}^{i}
\end{array}\right. \\
\left\{\begin{array}{c}
e_{n}^{i}=q_{n}^{i}(\alpha) q_{n}^{i}(1-\alpha)\left(q_{n-2}^{i}(\alpha) q_{n-1}^{i}(\alpha)+q_{n-2}^{i}(1-\alpha) q_{n-1}^{i}(1-\alpha)\right) \\
f_{n}^{i}=q_{n-2}^{i}(\alpha) q_{n-2}^{i}(1-\alpha)\left(q_{n-1}^{i}(\alpha) q_{n}^{i}(\alpha)+q_{n-1}^{i}(1-\alpha) q_{n}^{i}(1-\alpha)\right)
\end{array}\right.
\end{gathered}
$$

By Theorem 3.5, we deduce that $\frac{p_{n}^{i}}{q_{n}^{i}}$ converges to $\lambda_{i}^{\alpha}+\lambda_{i}^{1-\alpha}$ for $1 \leq i \leq m$. It follows that the matrix $\frac{P_{n}}{Q_{n}}$ converges to $D^{\alpha}+D^{1-\alpha}$. So, we get

$$
D^{\alpha}+D^{1-\alpha}=\left[2 I ; \frac{D_{1}+\widetilde{D}_{1}}{D_{1} \widetilde{D}_{1}}, \frac{D_{1} \widetilde{D}_{1} F_{2}^{\prime}}{E_{2}^{\prime}-F_{2}^{\prime}}, \frac{E_{n-1}^{\prime} F_{n}^{\prime}}{E_{n}^{\prime}-F_{n}^{\prime}}\right]_{n=3}^{+\infty}
$$

By Proposition 2, we have

$$
A^{\alpha}+A^{1-\alpha}=X\left(D^{\alpha}+D^{1-\alpha}\right) X^{-1}=\left[2 I ; \frac{\left(D_{1}+\widetilde{D}_{1}\right) X^{-1}}{D_{1} \widetilde{D}_{11} X^{-1}}, \frac{\left(D_{1} \widetilde{D}_{1}\right) F_{2}^{\prime} X^{-1}}{E_{2}^{\prime}-F_{2}^{\prime}}, \frac{E_{n-1}^{\prime} F_{n}^{\prime}}{E_{n}^{\prime}-F_{n}^{\prime}}\right]_{n=3}^{+\infty}
$$

Let us define the sequence $\left.\left(X_{n}\right)\right)_{n \geq-1}$ by $X_{-1}=X_{0}=I$, and for all $n \geq 1, X_{n}=X$. Then we have

$$
\left\{\begin{array}{c}
\frac{X_{1}\left(D_{1}+\widetilde{D}_{1}\right) X^{-1} X_{-1}^{-1}}{X_{1} D_{1} D_{1} \widetilde{D}_{1} X^{-1} X_{0}^{-1}}=\frac{A_{1}+\widetilde{A}_{1}}{A_{1} \widetilde{A}_{1}} \\
\frac{X_{2}\left(D_{1} D_{1} \widetilde{D}_{1} F_{2}^{\prime} X^{-1}\right) X_{0}^{-1}}{X_{2}\left(E_{2}^{\prime}-F_{2}^{\prime}\right) X_{1}^{-1}}=\frac{A_{1} \widetilde{A}_{1} F_{2}}{E_{2}-F_{2}} \\
\frac{\left.X_{n} E_{n-1}^{\prime} F_{n}^{\prime}\right) X_{n-2}^{-1}}{X_{n}\left(E_{n}^{\prime}-F_{n}^{\prime}\right) X_{n-1}^{-1}}=\frac{E_{n-1} F_{n}}{E_{n}-F_{n}}
\end{array}\right.
$$

with $X E_{n}^{\prime} X^{-1}=E_{n}$ and $X F_{n}^{\prime} X^{-1}=F_{n}$ for all $n \geq 2$.

By applying the result of Proposition 3 to the sequence $\left(X_{n}\right)_{n}$, we finish the proof of Theorem 1 . 


\subsection{Expansion of the Operator of Heinz}

The original motivation of this article was the problem of representation the Heinz operator in continued fraction in order to make its computation practical and efficient. Given two positive definite matrices $A, B$ we recall that the Heinz operator mean is the map

$$
H_{\alpha}(A, B)=A^{\frac{1}{2}} \frac{\left(A^{\frac{-1}{2}} B A^{\frac{-1}{2}}\right)^{\alpha}+\left(A^{\frac{-1}{2}} B A^{\frac{-1}{2}}\right)^{1-\alpha}}{2} A^{\frac{1}{2}}
$$

Theorem 3.6. Let $A, B \in \mathcal{M}_{m}$ be two positive definite matrices, $\alpha$ a positive real number such that $0<\alpha<1$. A continued fraction expansion of $H_{\alpha}(A, B)$ is

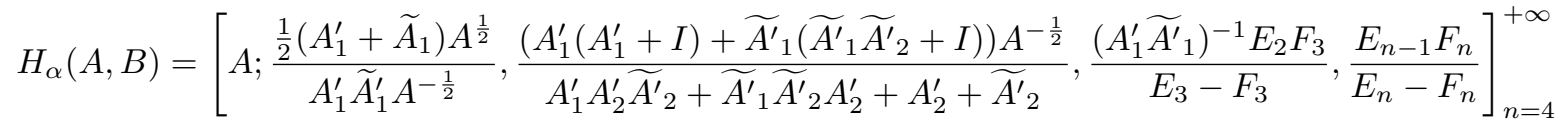

where

$$
\left\{\begin{array}{l}
A_{1}^{\prime}=\frac{-I-\alpha \phi\left(A^{-\frac{1}{2}} B A^{-\frac{1}{2}}\right)}{2 \alpha \phi\left(A^{-\frac{1}{2}} B A^{-\frac{1}{2}}\right)}=\frac{-I-\alpha A^{\frac{1}{2}}\left(\frac{A-B}{A+B}\right) A^{\frac{-1}{2}}}{2 \alpha A^{\frac{1}{2}}\left(\frac{A-B}{A+B}\right) A^{\frac{-1}{2}}}, \\
A_{2}^{\prime}=\frac{-10 \alpha\left(\frac{A-B}{A+B}\right)^{2} A^{\frac{-1}{2}}}{\left(\alpha^{2}-1\right)\left(\frac{A-B}{A+B}\right)^{2} A^{\frac{-1}{2}}} .
\end{array}\right.
$$

The expressions of $\widetilde{A^{\prime}}{ }_{1}$ and $\widetilde{A^{\prime}}{ }_{2}$ are obtained from that of $A_{1}^{\prime}$ and $A_{2}^{\prime}$ respectively by replacing $\alpha$ by $(1-\alpha)$. The matrices $E_{n}$ and $F_{n}$ are the same as in Theorem 1 .

We deduce a continued fraction expansion of the symmetric operator entropy $S T_{\alpha}(A, B)$.

Corollary 3.7. Let $A, B \in \mathcal{M}_{m}$ be two positive definite matrices, $\alpha$ a positive real number such that $0<\alpha<1$. With the same notations as in Theorem 3, a continued fraction expansion of $S T_{\alpha}(A, B)$ is

$$
S T_{\alpha}(A, B)=\left[\frac{A-B}{2 \alpha} ; \frac{\frac{1}{2 \alpha}\left(A_{1}^{\prime}+\widetilde{A_{1}}\right) A^{\frac{1}{2}}}{A_{1}^{\prime} \widetilde{A^{\prime}}{ }_{1} A^{-\frac{1}{2}}}, \frac{\left(A_{1}^{\prime}\left(A_{1}^{\prime}+I\right)+\widetilde{A^{\prime}}{ }_{1}\left(\widetilde{A^{\prime}}{ }_{1} \widetilde{A_{2}^{\prime}}+I\right)\right) A^{-\frac{1}{2}}}{\left.A_{1}^{\prime} A_{2}^{\prime} \widetilde{A_{2}^{\prime}}+\widetilde{A^{\prime}}{ }_{1} \widetilde{A^{\prime}}{ }_{2} A_{2}^{\prime}+A_{2}^{\prime}+\widetilde{A_{1}^{\prime}}{ }_{2}{\widetilde{A^{\prime}}}_{1}\right)^{-1} E_{2} F_{3}} \frac{E_{n-1} F_{n}}{E_{3}-F_{3}}\right]_{n=4}^{+\infty}
$$

Proof of Theorem 3.5. Since

$$
\left(A^{\frac{-1}{2}} B A^{\frac{-1}{2}}\right)^{\alpha}+\left(A^{\frac{-1}{2}} B A^{\frac{-1}{2}}\right)^{1-\alpha}=2 A^{\frac{-1}{\amalg} 2} H_{\alpha}(A, B) A^{\frac{-1}{2}},
$$

According to Theorem 1, the continued fraction expansion of $H_{\alpha}(A, B)$ is

$$
H_{\alpha}(A, B)=\frac{1}{2} A^{\frac{1}{2}}\left[2 I ; \frac{A_{1}^{\prime}+\widetilde{A_{1}}}{A_{1}^{\prime} \widetilde{A^{\prime}}{ }_{1}}, \frac{A_{1}^{\prime}\left(A_{1}^{\prime}+I\right)+\widetilde{A^{\prime}}{ }_{1}\left(\widetilde{A^{\prime}}{ }_{1} \widetilde{A^{\prime}}{ }_{2}+I\right)}{A_{1}^{\prime} A_{2}^{\prime} \widetilde{A^{\prime}}{ }_{2}+\widetilde{A^{\prime}}{ }_{1}{\widetilde{A^{\prime}}}_{2}{ }_{2} A_{2}^{\prime}+A_{2}^{\prime}+\widetilde{A^{\prime}}{ }_{2}}, \frac{\left(A_{1}^{\prime} \widetilde{A^{\prime}}{ }_{1}\right)^{-1} E_{2} F_{3}}{E_{3}-F_{3}}, \frac{E_{n-1} F_{n}}{E_{n}-F_{n}}\right]_{n=4}^{+\infty}
$$

By applying the result of Proposition 2, we obtain

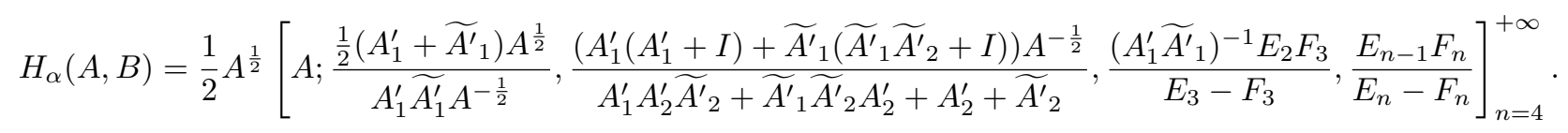

Remark 2. The matrices $E_{n}$ and $F_{n}$ are the same as in Theorem 3.1.

Which completes the proof of Theorem 3.6.

\section{Conclusion}

Many researchers have studied a various means inequalities, the Heinz norm inequalities for positive operators in Hilbert spaces. In this work, we provide a method which allows us to compute the Heinz operator mean $H_{\alpha}(A, B)$ of two positive definite matrices $A$ and $B$. It is defined by

$$
H_{\alpha}(A, B)=A^{\frac{1}{2}} \frac{\left(A^{\frac{-1}{2}} B A^{\frac{-1}{2}}\right)^{\alpha}+\left(A^{\frac{-1}{2}} B A^{\frac{-1}{2}}\right)^{1-\alpha}}{2} A^{\frac{1}{2}},
$$

where $\alpha$ is a positive real number such that $0<\alpha<1$.

In Theorem 3.6, a continued fraction expansion of the Heinz operator mean $H_{\alpha}(A, B)$ is given.

As a corollary of this result, an algorithm of calculus of the symmetric operator entropy

$$
S T_{\alpha}(A, B)=\frac{1}{\alpha}\left(H_{\alpha}(A, B)-\frac{A+B}{2}\right) .
$$


is also presented.

Our contribution allowed us to avoid the computation of these operators by the exponential and logarithm functions of matrices.

It should be noted that we can use the Padé approximation in order to compute the Heinz opertor mean.

\section{References}

[1] T. ANDO, Topics on operators inequalities, Ruyuku Univ. Lecteure Note Series, 1 (1978).

[2] M. ALAKHRASS, M. SABABHEH, Matrix mixed mean inequalities. Results Math. 74, no 1 (2019), Art 213.

[3] A. A. BURQAN, Comparisons of Heinz Operator Parameters. Malaysian Journal of Science, Vol. 38, S1, (2019), 33-42

[4] S. S. DRAGOMIR, Some inequalities for Heinz operator mean, Mathematica Moravica, vol. 24, no 1 (2020), 7182.

[5] T. H. DINH, R. DUMITRU, J. A. FRANCO, THe matrix power mean and interpolations. Adv. Oper. Theory 3 (2018), 647-654.

[6] B. W. HELTON, Logarithms of matrices, Proc. Amer. Math. Scoc., 19 (1968), 733-738.

[7] F. R. GANTMACHER, The Theory of matrices, Vol. I. Chelsa. New York, Elsevier Science Publiscers, (1992).
[8] Y. KAPIL, C. CONDE, M. S. MOSLEHIAN, M. SABABHEH and M. SING. Norm inequalities related to the Heron and Heinz means. Mediterr. J. Math. 14, (2017), Art. 213.

[9] A. N. KHOVANSKI, The applications of continued fractions and their Generalisation to problemes in approximation theory, (1963), Noordhoff, Groningen, The Netherlands.

[10] L. LORENTZEN, H. WADELAND, Continued fractions with applications, Elsevier Science Publishers, 1992.

[11] G. J. MURPHY, $C^{*}$-Algebras and operators theory, Chapter 2, (1990), Academic press, INC Harcourt Brace Jovanovich, publishers.

[12] N. NEGOESCU, Convergence theorems on noncommutative continued fractions, Rev. Anal. Numé. Thérie Approx., 5 (1977), 165-180.

[13] G. NETLLER, On trenscendental numbers whose sum, difference, quotient and product are transcendental numbers, Math. Student 41, No. 4 (1973), 339-348.

[14] M. RAISSOULI, A. KACHA, Convergence for matrix continued fractions, Linear Algebra Appl., 320 (2000), 115-129.

[15] M. RAISSOULI, A. KACHA, S. SALHI, The Arabian Journal for Science and Engineering, Volume 31, Number 1 A (2006), 1-15.

[16] M. SABABHEH, Convexity and matrix means. Linear Algebra Appl. 506 (2016), 588-602. 